\title{
Fasting and Refeeding Affect the Goose Liver Transcriptome Mainly Through
}

\section{the PPAR Signaling Pathway}

Zhenzhen $\mathrm{Chen}^{1}$, Ya Xing ${ }^{1}$, Xue Fan ${ }^{1}$, Tongjun Liu ${ }^{1}$, Minmeng Zhao ${ }^{1}$, Long Liu ${ }^{1}$, Xuming $\mathrm{Hu}^{1}$, Hengmi $\mathrm{Cui}^{1}$, Tuoyu Geng ${ }^{1,2}$ and Daoqing Gong ${ }^{1,2}$

${ }^{1}$ College of Animal Science and Technology, Yangzhou University, Yangzhou 225009, Jiangsu Province, P. R. China

${ }^{2}$ Joint International Research Laboratory of Agriculture and Agri-Product Safety of the Ministry of Education of China, Yangzhou University, Yangzhou 225009, Jiangsu Province, P. R. China

Running title: Nutritional status affects lipid metabolism

Correspondence: Tuoyu Geng and Daoqing Gong

College of Animal Science and Technology, Yangzhou University, 48 Wenhui East

Road, Yangzhou, Jiangsu Province 225009, P. R. China

Email: tygeng@yzu.edu.cn (Tuoyu Geng); yzgong@163.com (Daoqing Gong) 


\section{Abstract}

Nutrition and energy are essential for poultry growth and production performance.

Fasting and refeeding have been widely used to study the effects of nutrition, energy, and related mechanisms in chicken. Previous studies have shown that geese have a strong capacity for fat synthesis and storage; thus, changes in the goose liver transcriptome may be different from those in chicken assessed with a model of fasting and refeeding. However, the responses of the goose liver transcriptome to fasting and refeeding have not yet been addressed. In this study, 36 70-day-old Si Ji geese with similar body weight were randomly assigned to three groups: control (ad libitum feeding), fasting (fasted for $24 \mathrm{~h}$ ), and refeeding (fast for $24 \mathrm{~h}$ followed by 2 -h feeding) groups. After treatment, eight geese per group were sacrificed for sample collection. Liver samples from four geese in each group were subjected to transcriptome analysis, followed by validation of differentially expressed genes (DEGs) using quantitative polymerase chain reaction with the remaining samples. As a result, 155 DEGs (73 up-regulated) were identified between the control and fasting groups, and 651 DEGs (321 up-regulated) were identified between the fasting and refeeding groups. The enrichment analyses of Gene Ontology terms and Kyoto Encyclopedia of Genes and Genomes pathways showed that fasting mainly influenced material metabolism in the liver, especially lipid metabolism; in contrast, refeeding affected not only lipid metabolism but also glucose and amino acid metabolism. In addition, the peroxisome proliferator-activated receptor (PPAR) signaling pathway may play an important role in lipid metabolism. In conclusion, fasting and refeeding have a strong effect on lipid 
metabolism in the goose liver; specifically, fasting promotes fatty acid oxidation and inhibits fatty acid synthesis, and refeeding has the opposite effect. The model of fasting and refeeding is suitable for goose nutrition studies.

Key words: goose, lipid metabolism, liver, nutrition, transcriptome analysis 


\section{Introduction}

The growth and development of animals require a continuous supply of nutrition and energy, and the nutrition and energy needs of animals differ according to different life stages. For example, laying hens need to be fed appropriately during the late stages of laying. A diet that provides more nutrition and energy than needed by hens could lead to the development of fatty liver hemorrhage syndrome. By contrast, geese should be overfed to allow for the rapid development of fatty liver. Evidence indicates that nutritional factors have a strong impact on animal growth, reproduction, and immunity. Many metabolism-related diseases (e.g., diabetes, non-alcoholic fatty liver disease, metabolic syndrome, and other obesity-related metabolic diseases) are also closely related to nutritional and energy problems (Streba et al., 2008; American Diabetes Association, 2014; Buzzetti et al., 2016). Carbohydrates, proteins, fats, vitamins, minerals, and other nutrients are not only the raw materials for building cells but some of these nutrients and their derivatives can also act as signal molecules (e.g., sphingolipids) to participate in signaling regulation, thus influencing gene expression and cell function.

Fasting (or starvation) and feeding are two completely different nutritional statuses. Fasting can cause a slump in nutrition and energy in animals, leading to a switch in energy supply from glucose metabolism to fat metabolism. Refeeding has the opposite effect, which can cause a dramatic increase in nutrition and energy, leading to the rapid elevation of blood glucose, and an increase in fat synthesis and storage. Previous studies have indicated that fasting, even for a short period of time, 
can dramatically reduce the capacity of lipogenesis in the chicken liver, whereas refeeding for $1 \mathrm{~h}$ can restore the reduced capacity (Leveille et al., 1975). Thus, identifying the drastic changes in nutrition and energy levels caused by fasting and refeeding may facilitate gaining an in-depth understanding of the molecular mechanisms by which nutrition and energy metabolic processes affect animal physiological functions and disease development.

Existing evidence shows that fasting and feeding can modulate gene expression and signal transduction in chickens. For example, Wang et al. (2020) showed that fasting activated AMP-activated protein kinase (AMPK), leading to inhibition of glycogen synthase, fatty acid synthase $(F A S N)$, and sterol regulatory element binding protein 1 (SREBP 1) expression, and induction of the downstream gene glucose-6-phosphatase catalytic subunit 2 in the liver of 20-day-old chickens. Fujita et al. (2018) reported that $6 \mathrm{~h}$ of fasting significantly increased the mRNA expression levels of insulin-like growth factor binding protein-1 and 2 in the livers of 8-day-old chickens, which could be reversed by $6 \mathrm{~h}$ of refeeding. Li et al. (2011) found that fasting for 24 or $48 \mathrm{~h}$ induced the mRNA expression of F-box protein 32 and tripartite motif-containing 63 in the skeletal muscle of 7-day-old chicks, and refeeding suppressed this induction. Based on transcriptome analysis, Ji et al. (2012) identified 2016 differentially expressed genes (DEGs), including 917 up-regulated and 863 down-regulated genes, in the fasted vs. fed adipose tissue of 17-day-old commercial broiler chickens from three groups (i.e., chickens fed ad libitum, fasted for $5 \mathrm{~h}$, and those fed but deprived of insulin by injections of anti-insulin serum). These DEGs 
were enriched in a broad series of metabolism, signaling, and adipogenesis pathways such as those related to sphingolipid metabolism, peroxisome proliferator-activated receptor (PPAR) signaling, fatty acid metabolism, and the peroxisome. Desert et al. (2018) performed transcriptome analysis on the livers of 4-week-old broiler chickens that were fasted for 16 or $48 \mathrm{~h}$ and those fed ad libitum, and identified 1162 DEGs; up-regulated genes in the fasted group were associated with fatty acid oxidation, ketogenesis, and gluconeogenesis, whereas the down-regulated genes were associated with fatty acid and cholesterol synthesis. In contrast to other transcriptomic studies that mainly focused on juvenile chickens (1, 2, or 4 weeks old), Cogburn et al. (2020) carried out transcriptome analysis on the livers of newly hatched chicks that were fasted for 4,24 , or $48 \mathrm{~h}$ in comparison to those of fully fed chicks. They identified several highly expressed upstream regulators, including those related to the fasting-lipolytic state (PPARA, NR3C1, NFE2L2, SERTAD2, FOX01, NROB1, and $R X R$ ) and those related to the fully fed lipogenic/thermogenic state (THRSPA, SREBF2, PPARG, PPARD, JUN, ATF3, and CTNNB1). Accordingly, the downstream genes, including the lipogenic genes (e.g., FASN, ME1, $S C D$ ) and lipolytic genes (e.g., $A L D O B, L D H B, L P I N 2)$ were down-regulated and up-regulated by fasting, respectively, and these changes could be reversed by refeeding (Cogburn et al., 2020). In summary, these studies provided deep insight into the mechanisms by which energy/material metabolism is affected by fasting and feeding in chickens.

Compared to chickens, the effects of fasting and refeeding have barely been investigated in geese. Previous studies have indicated that the goose, as a descendant 
of a migrant bird, has an excellent capacity to deposit fat in the liver. For example, the goose liver can grow to be 8-10-times heavier (about 800-1200 g) than the normal liver after a short period (3-4 weeks) of artificial overfeeding, and the fat content in this overfed liver can reach up to $\sim 60 \%$ (Fournier et al., 1997). This unique feature suggests that goose lipid metabolism may differ from that of chicken in some respects. Therefore, this study was designed to investigate the effects of fasting and refeeding on the liver transcriptome of geese, and to screen for DEGs and associated enriched signaling pathways. These findings may provide a foundation for studying the mechanisms by which nutritional/energy levels affect production performance and some metabolism-related diseases (such as fatty liver) in geese.

\section{Materials and Methods}

\section{Experimental Animals and Sample Collection}

All animal protocols were approved by the Animal Care and Use Committee of Yangzhou University (IACUC certificate number NSFC2020-DKXY-22). Thirty-six healthy 1-day-old Si Ji geese were purchased from Jiangsu Si Ji Goose Breeding Company (Jurong, Jiangsu, China) and raised in Mali Agricultural Ecological Park (Jurong, Jiangsu, China). During the brooding period, the goslings were raised on a net with $24 \mathrm{~h}$ lighting per day and the temperature maintained at $28-30^{\circ} \mathrm{C}$ with infrared lamps. The goslings had free access to feed and water. The formula for the brooding diet is presented in Supplementary Table 1. At 28 days of age, the geese were raised on a net under natural temperature and lighting conditions until the age of 70 days. The geese also had free access to feed and water. The formula for the 
growing diet is shown in Supplementary Table 2. At 71 days old, 24 healthy geese with similar body weight were randomly assigned into three groups (eight geese per group): the control group (average body weight $4.18 \pm 0.13 \mathrm{~kg}$, ad libitum feeding for $24 \mathrm{~h}$ from $12 \mathrm{PM}$ to $12 \mathrm{PM}$ ), fasting group (average body weight $4.14 \pm 0.11 \mathrm{~kg}$, fasted for $24 \mathrm{~h}$ from $12 \mathrm{PM}$ to $12 \mathrm{PM}$ ), and refeeding group (average body weight $4.13 \pm 0.12 \mathrm{~kg}$, fasted for $24 \mathrm{~h}$ followed by refeeding for $2 \mathrm{~h}$ from $10 \mathrm{AM}$ to $12 \mathrm{PM}$ the next day). All geese were sacrificed within a short period $(<15 \mathrm{~min})$ before the liver samples were harvested. Four liver samples from each group were used for the transcriptome sequencing analysis. Eight liver samples from each group were used for quantitative polymerase chain reaction (qPCR) verification of the expression levels of the DEGs identified by transcriptome analysis.

Extraction and Purification of Total RNA, Reverse Transcription, and qPCR

\section{Analysis}

Total RNA was extracted from the liver samples using TRIzol (Cat. No. DP424, Tiangen Biotech Co., Ltd., Beijing, China), HiScript TMQ RT Super Mix Reverse Transcription kit (Cat\# R123-01, Vazyme Biotech Co., Ltd., Nanjing, China) was used to synthesize the first-strand cDNA, and the Vazyme AceQTM qPCR SYBR Green Master Mix kit (Cat\# Q111-02/03, Vazyme Biotech Co., Ltd., Nanjing, China) was used for fluorescence qPCR analysis. All procedures were performed according to the manufacturers' instructions. Using the online software Primer 3.0 (http://bioinfo.ut.ee/primer3-0.4.0/), primers were designed according to the mRNA sequences of genes of interest retrieved from GenBank or from our local database 
established based on previous sequencing analysis of the goose liver transcriptome. The binding specificity of the primers was checked using the National Center for Biotechnology Information Primer-Blast online program. The glyceraldehyde phosphate dehydrogenase $(G A P D H)$ gene and $\beta$-actin gene were used as internal reference genes. The primer sequences are listed in Table 1.

\section{Transcriptome Sequencing Analysis}

The quantity and quality of total RNA samples were analyzed using a Qubit 2.0 fluorometer (ThermoFisher Scientific Inc.) and Agilent 2100 Bioanalyzer (Agilent Technologies), respectively. The cDNA library was subsequently constructed by reverse transcription using qualified total RNA samples. In brief, eukaryotic mRNA samples were enriched with Oligo (dT) bound to magnetic beads, followed by random interruption of mRNA in fragmentation buffer. The first strand of cDNA was then synthesized by reverse transcription with six-base random primers (random hexamers), and the second strand of cDNA was synthesized by adding reaction buffer, dNTPs, RNase $\mathrm{H}$, and DNA polymerase I to the previous solution. cDNA samples were purified using AMPure XP beads. After repairing the ends of cDNA, connecting with sequencing adapters, and selecting the appropriate size of fragments with AMPure XP beads, a cDNA library was constructed by PCR enrichment. The quality of the cDNA library was then checked and sequenced on an Illumina high-throughput sequencing platform (HiSeq X-ten) using sequencing-by-synthesis technology.

Clean data with a quality score of Q30 or more were selected for further analysis according to the calculated Phred score on a large amount of raw data. By aligning 
against the sequence of the designated reference genome, clean data were annotated using the HISAT2 system. Single genes (i.e., unigenes) were assembled by comparing the reads with the StringTie program. By standardizing the reads to units of fragments per kilobase of transcript per million fragments mapped (FPKM), the expression level of a single gene was calculated. On this basis, DEGs were identified by comparing the expression level of each gene between the different groups. The criteria for a DEG were fold change $\geq 2$ or $\leq 0.5$ and false discovery rate $(\mathrm{FDR})<0.05$. With the identified DEGs, Gene Ontology (GO) function and enrichment analyses were performed using the Kolmogorov-Smirnov analysis method in the top GO R data package (the standard for significant enrichment was set to a KS-value $<0.05$ ). Enrichment analysis of Kyoto Encyclopedia of Genes and Genomes (KEGG) pathways was performed using the KOBAS program (version 2.0). The standard of significant enrichment for KEGG pathway analysis was set as a q-value $<0.05$.

\section{Statistical Analysis}

The results from the qPCR analysis were calculated using the $2^{-\triangle \Delta \mathrm{Ct}}$ method. All data are expressed as the mean \pm SEM. One-way analysis of variance in SPSS software (version 16.0) was used to evaluate the statistical significance of the differences among groups, followed by pairwise comparisons using Tukey's post-hoc test. $P<0.05$ and $P<0.01$ were judged as significant and extremely significant differences, respectively. 


\section{Results}

\section{Identification of DEGs in the Goose Liver Transcriptome}

By sequencing the transcriptomes of four liver samples from each group, a total of 98.19 Gb clean data was obtained, and the clean data across the samples reached an average of $6.71 \mathrm{~Gb}$. The Q30 base percentage was above 93.93\%, the mapping efficiency of clean reads to the reference genome was 76.48-81.41\% (Supplementary Table 3), and a total of 15,517 genes were annotated (Supplementary Table 4). DEGs were identified by comparing the expression level of each gene between groups. The heat map of all DEGs indicated that the samples for sequencing had good repeatability (Supplementary Fig. 1). Compared with the control group, the fasting group had 155 DEGs (73 up-regulated and 82 down-regulated; Supplementary Table 5). Compared with the fasting group, the refeeding group had 651 DEGs (321 up-regulated and 330 down-regulated; Supplementary Table 6). Among the DEGs, 94 were common to the fasting and refeeding groups. The 10 most significant (or Top 10) up-regulated and down-regulated DEGs according to the FDR are listed in Tables 2 and 3 , respectively.

\section{GO Functional Annotation and Enrichment Analysis}

A total of 72 DEGs in the fasting group vs. the control group and 271 DEGs in the refeeding group vs. the fasting group were annotated for GO functional terms. The DEGs enriched in the secondary tier of functional GO categories are shown in Supplementary Fig. 2A and Supplementary Fig. 2B. Notably, in the secondary functional tier "metabolic process" of the first-tier biological process category, the 
enrichment ratio of DEGs (62.5\%) in the fasting group vs. the control group was $24.2 \%$ higher than that of all annotated genes (38.3\%), whereas the enrichment ratio of DEGs (43.9\%) in the refeeding group vs. the fasting group was $5.6 \%$ higher than that of all annotated genes (38.3\%). In the secondary functional tiers "extracellular region" and "extracellular region part" of the first-tier cellular component category, the enrichment ratios of DEGs in the fasting group vs. the control group (12.5\% and $9.7 \%)$ were $5.4 \%$ and $5.1 \%$ higher than those of all annotated genes $(7.1 \%$ and $4.6 \%)$, respectively. In contrast, the enrichment ratio of DEGs in the overfeeding group vs. the fasting group had no secondary functional tiers that were at least 5.0\% higher than the enrichment ratio of all annotated genes. In the secondary functional tier "catalytic activity" of the first-tier molecular function category, the enrichment ratio of DEGs in the fasting group vs. the control group (59.7\%) was $24.9 \%$ higher than that of all annotated genes (34.9\%). Similarly, the enrichment ratio $(45.0 \%)$ of DEGs in the overfeeding group vs. the fasting group was $10.2 \%$ higher than that of all annotated genes $(34.9 \%)$.

There were 356 and $361 \mathrm{GO}$ terms between the fasting group and the control group, and between the refeeding group and the fasting group, respectively, with a significant difference detected between the actual and estimated numbers of DEGs $(\mathrm{KS}<0.05)$. Among these GO terms, 81 and 131 terms were significantly enriched (i.e., the actual number of DEGs was significantly higher than the estimated number of DEGs; Supplementary Tables 7 and 8). There were 21 DEGs involved in the GO terms that were significantly enriched between the fasting group and the control group, 
including HMGCS2, PCK1, SOD1, CPT1A, HADHB, HADHA, LPIN1, ACSL5, $C T G F, L I P C, A C O X 1, A B H D 2, P D K 3, L D H B$, and SLC25A33. There were 66 DEGs involved in GO terms that were significantly enriched between the refeeding and fasting groups, including PANK1, HMGCS2, PCK1, HSD17B11, SOD1, ACACA, SLC25A33, CPT1A, FASN, HADHB, HADHA, ACOX1, LIPC, ACSL5, ME1, and $L D H B$. In the comparison of the fasting group and the control group, 31 of the significantly enriched GO terms were related to lipid metabolism (e.g., cholesterol biosynthetic process, triglyceride metabolic process, fatty acid metabolic process, very-low-density lipoprotein particle remodeling, 3-hydroxyacyl-CoA dehydrogenase activity), mitochondria (e.g., mitochondrial inner membrane, mitochondrial outer membrane, mitochondrial pyruvate transport), and oxidative stress (e.g., superoxide dismutase activity and superoxide anion generation) (Table 4). In the comparison of the refeeding and fasting groups, 30 significantly enriched GO terms were also related to lipid metabolism, mitochondria, and oxidative stress (Table 5). These GO terms were similar to those identified in the comparison of the fasting and control groups.

\section{Enrichment Analysis of KEGG Pathways}

A total of 70 DEGs between the control group and the fasting group were enriched in 29 KEGG pathways (Supplementary Table 9). The top 20 KEGG pathways with the highest enrichment significance (Fig. 1A) and the DEGs involved in these pathways are listed in Table 6. Among the pathways, there were at least seven pathways closely related to fat metabolism, including fatty acid metabolism (the ratio of the number of DEGs enriched in the KEGG pathway to the number of all DEGs 
was $20.0 \%$ ), fatty acid degradation (17.14\%), PPAR signaling pathway (12.86\%), biosynthesis of unsaturated fatty acids (8.57\%), synthesis and degradation of ketone bodies (5.71\%), fatty acid biosynthesis (5.71\%), and adipocytokine signaling pathway (8.57\%). The DEGs involved in these pathways included EHHADH, PCK1, CPT1A, $C P T 1 B, A C S L 5, A C O X 1, A C A A 1, S C D$, and $A C S B G 2$. There were at least three pathways closely related to amino acid/protein metabolism, including valine, leucine and isoleucine degradation (12.86\%); tryptophan metabolism (10\%); and arginine and proline metabolism (5.71\%). The DEGs involved in these pathways included STC2 and AZIN2. There were at least two pathways closely related to glucose metabolism, including pyruvate metabolism (10\%) and glycolysis/gluconeogenesis $(8.75 \%)$, and the DEGs involved in these pathways included PCK1, ACAT1, LDHB, ACSS2, and ME1.

Similarly, 218 DEGs between the fasting and refeeding groups were enriched in 31 pathways (Supplementary Table 10). The top 20 KEGG pathways with the highest enrichment significance (Fig. 1B) and the associated DEGs are listed in Table 7. Among these pathways, at least seven pathways were closely related to fat metabolism, including the adipocytokine signaling pathway (5.96\%), PPAR signaling pathway (5.96\%), fatty acid metabolism (5.05\%), fatty acid biosynthesis (2.75\%), biosynthesis of unsaturated fatty acids (3.67\%), fatty acid elongation (2.29\%), and fatty acid degradation (2.75\%). The DEGs involved in these pathways included ACSBG2, IRS1, SCD, FABP7, ACACA, FASN, ELOVL5, IRS4, PCK1, CPT1A, G6PC, $A C A C B, A C S L 5, P P A R G C 1 A, A C S L 1, P R K A G 2$, and $A K T 1$. At least six pathways 
were closely related to glucose metabolism, including the insulin signaling pathway $(8.72 \%)$, starch and sucrose metabolism (4.59\%), FoxO signaling pathway $(6.88 \%)$, amino sugar and nucleotide sugar metabolism (2.75\%), galactose metabolism (2.29\%), and glycolysis/gluconeogenesis (3.21\%). The DEGs involved in these pathways included PPP1R3C, CRK, PIK3R1, ACACA, GINS4, FASN, IRS1, PGM2, UGDH, GBE1, UGP2, CCNB1, SGK1, IGF1, GFPT1, PCK1, G6PC, PGM3, IRS4, ACACB, PPARGC1A, PRKAG2, AKT1, and GYS2. There were at least three pathways closely related to amino acid/protein metabolism, including alanine, aspartate, and glutamate metabolism $(2.29 \%)$ and protein processing in the endoplasmic reticulum $(6.42 \%)$. The DEGs involved in these pathways included STC2, HGD, AFMID, TGM2, SH3RF1, LOC106032502, and AZIN2. The critical pathways with differences in the control vs. fasting and fasting vs. refeeding comparisons (selected from the top 20 KEGG pathways) are listed in Supplementary Table 11.

In addition, fluorescent $\mathrm{qPCR}$ with $G A P D H$ and $\beta$-actin as internal reference genes was used to verify the differential expression of nine DEGs involved in lipid metabolism identified from the transcriptome analysis, with a focus on those involved in the PPAR signaling pathway: ACSBG2, ACOX1, CPT1A, ACSL5, ACAA1, CPT1B, EHHADH, SCD, and PCK1. Among these nine genes, the qPCR results showed that the trends in the expression of eight DEGs were consistent with the results from the transcriptome sequencing analysis, with only an inconsistent trend found in the expression of $C P T 1 B$ (Fig. 2A, 2B, Supplementary Fig. 3). These results indicated the overall good reliability of the transcriptome sequencing analysis. 


\section{Discussion}

The liver is an important organ for the metabolism and distribution of nutrition in animals, and its transcriptome can be easily altered by variations in nutrition and energy sources. The liver serves as the main site for fatty acid synthesis in avian species as opposed to the adipose tissue playing this role in mammals (Goodridge, 1968; O'Hea and Leveille, 1969; Yeh and Leveille, 1973); thus, the avian liver may play a more important role in lipid metabolism. In mammals, lipid metabolism is regulated by PPARa, PPAR $\gamma$, SREBP1, SREBP2, and NR1H3, which are critical transcription factors for the regulation of fatty acid beta-oxidation, fatty acid synthesis, and cholesterol metabolism (Shchelkunova et al., 2013). Similarly, previous studies have indicated that PPAR $\alpha$ plays a key role in the up-regulation of fatty acid beta-oxidation, whereas PPAR $\gamma$ is the key regulator of fatty acid synthesis in the chicken (Désert et al., 2008; Navidshad and Royan, 2015). Consistently, Désert et al. (2008) identified a potential PPAR $\alpha$ response element located in $A C O X 1, C P T 1$, and $H M G C S 2$, and the mRNA expression levels of $A C O X 1$ and $C P T 1$ were significantly associated with that of PPAR $\alpha$. Moreover, SREBP1 is an important transcription factor involved in lipogenesis, whereas SREBP2 regulates cholesterol synthesis. Désert et al. (2008) also observed a significant correlation between SREBP1 mRNA levels and those of its putative target genes $A C L Y, A C A C A, F A S N$, and $S C D$, which are known to be involved in lipogenesis. Compared to that in the chicken, there has been much less research focused on lipid metabolism in the goose liver. Considering the unique features of lipid metabolism in geese, it is uncertain whether these critical 
transcription factors also play an important role in the regulation of fatty acid beta-oxidation, fatty acid synthesis, and cholesterol metabolism in the goose liver.

Experimental chicken models of fasting and refeeding are widely used in research. We also used these models to identify the genes and pathways that were affected by fasting and refeeding in the livers of 70-day-old geese. When the geese were fasted for $24 \mathrm{~h}$, the changes in the liver transcriptome were mainly reflected in metabolic processes, especially in the pathways related to fat metabolism such as fatty acid degradation, the PPAR signaling pathway, biosynthesis of unsaturated fatty acids, and the synthesis and degradation of ketone bodies. The changes in these pathways were reflected by the up-regulated expression of CPT1A, ACAT1, CPT1B, HADHB, $H A D H A, A C S L 5, A C O X 1, A C A A 1$, and PCK1, and the down-regulated expression of $S C D, A C A C A$, and $F A S N$. This indicates an increase in fatty acid degradation and ketone body production in the goose liver during fasting. Among these pathways, the PPAR and SREBP signaling pathways may play an important role in the response of the goose liver to fasting and refeeding. This is based on the finding of induced expression of CPT1A, CPT1B, ACSL5, and ACOX1, the downstream genes of PPAR $\alpha$, whereas the expression of $S C D, A C A C A$, and $F A S N$, the downstream genes of SREBP, was inhibited by fasting, consistent with previous reports (Brandt et al., 1998; Yeon et al., 2004; Morais et al., 2007; Rakhshandehroo et al., 2007). Moreover, fatty acid oxidation is carried out in the mitochondrion, as the main site for the production of reactive oxygen species. Therefore, it is reasonable that the GO terms enriched with DEGs were not only related to fatty acid metabolism but also to mitochondria (e.g., 
mitochondrial inner membrane, mitochondrial outer membrane, mitochondrial pyruvate transport) and oxidative stress (e.g., superoxide dismutase activity and superoxide anion generation). The oxidative stress-related genes that were differentially expressed in a fasting condition included MPC1, SOD1, CPT1A, $H A D H B, H A D H A$, and $A C S L 5$. In addition, GO terms that regulate fat metabolism (e.g., cholesterol biosynthetic process) were enriched. Although the changes in the liver transcriptome in response to fasting were mainly related to fat metabolism, there were also some enriched pathways or GO terms related to amino acid, protein, and carbohydrate metabolism. Fat metabolism, protein metabolism, and carbohydrate metabolism all involve a series of enzyme catalytic reactions, which may explain why the DEGs were largely enriched in the secondary-tier GO function category "catalytic activity."

When an animal is refed for a period of time after fasting, the glucose level in the blood rises sharply, leading to the release of insulin, which in turn promotes the absorption of glucose by the liver and accelerates glucose conversion into fatty acids, most of which are transported to the adipose tissue for storage. In this study, the comparison between the refeeding and fasting groups indicated that the changes in the liver transcriptome were not only reflected in fat metabolism but also in the pathways related to carbohydrate metabolism. The majority of the fat metabolism-related pathways that were enriched after refeeding were those involved in fatty acid synthesis (e.g., fatty acid elongation), with fewer enriched pathways involved in the synthesis and degradation of ketone bodies, indicating a decrease in fatty acid 
oxidation and an increase in synthesis. The DEGs involved in these pathways included the down-regulated genes CPT1A, ACSL5, ACOX3, and ACSL1, as well as the up-regulated genes $F A S N, A C A C A$, and $S C D$. Consistently, the enriched GO terms included cholesterol biosynthetic process, triglyceride metabolic process, fatty acid metabolic process, very-low-density lipoprotein particle remodeling, and 3-hydroxyacyl-CoA dehydrogenase activity, all of which are related to the synthesis of fatty acids. In addition, GO terms that are associated with the regulation of fat metabolism (e.g., cholesterol biosynthetic process, regulation of cholesterol metabolic process) were also enriched after refeeding. Among the pathways related to carbohydrate metabolism, the insulin signaling pathway and FoxO signaling pathway may play important roles, characterized by the up-regulated genes $P P P 1 R 3 C, C R K$, PIK3R1, IRS1, SGK1, and IGF1, indicating that carbohydrate synthesis was significantly enhanced by refeeding.

It is interesting that the DEGs and enriched pathways identified in the refeeding vs. fasting model comparison partially overlapped with those identified in a previous goose overfeeding vs. normal feeding model comparison (Liu et al., 2016), especially those related to the metabolism of carbohydrates, lipids, and amino acids. However, the expression pattern of the DEGs identified in the refeeding vs. fasting and the overfeeding vs. normal-feeding comparisons was in contrast to that revealed by the fasting vs. normal-feeding model comparison. For example, overfeeding promoted fatty acid synthesis (as indicated by the up-regulated expression of GPI, PDH, CS, $A C L Y, A C C, F A S N, S C D$, and $D G A T 2$ ) and suppressed lipid packing and release (as 
indicated by the down-regulated expression of APOB26 and LPL27), whereas fasting promoted fatty acid oxidation and suppressed fatty acid synthesis as mentioned above. In addition, there are some striking differences between the overfeeding model and the fasting and refeeding models. In particular, in the later stage of overfeeding, the liver transcriptome was characterized by DEGs that were mainly enriched in the "cell growth and death" and "immune diseases" pathways, which were not revealed by the fasting and refeeding model. Overfeeding can induce dramatic growth of the goose liver, which leads to a fatty liver weight (about $800-1200 \mathrm{~g}$ ) that is 10-times heavier than the normal liver (approximately $80-120 \mathrm{~g}$ ). Overfeeding can also cause severe steatosis (about $60 \%$ fat in the liver) without any overt pathological symptoms, which suggests that geese may have a protective mechanism for preventing severe steatosis-associated inflammation and injury from a fatty liver. Therefore, the differences between the models are likely due to the dramatic cell growth, accompanied by suppressed inflammation and injury in the goose fatty liver. Indeed, the unique finding revealed by the overfeeding model was that complement genes, the key components of the immune response and inflammation, were entirely suppressed in the goose fatty liver compared with the normal liver.

In summary, when a goose is fasted, the response of the liver transcriptome is mainly related to fat metabolism; that is, fasting promotes fatty acid oxidation and ketone body synthesis, and inhibits fatty acid and cholesterol synthesis. When the goose is refed, the response of the liver transcriptome is not only related to fat metabolism (i.e., refeeding promotes fatty acid and cholesterol synthesis, and inhibits 
fatty acid oxidation and ketone body synthesis) but is also related to carbohydrate metabolism. These results are consistent with those of previous studies, indicating the feasibility of the fasting and refeeding model in goose nutritional studies. In addition, this study revealed the response of the goose liver transcriptome to drastic changes in nutritional status, and obtained a batch of DEGs with enriched GO terms and KEGG pathways. These data can therefore provide a good foundation for further investigations of the mechanism underlying the regulation of nutrition on animal growth and development, production performance, and disease occurrence.

\section{Acknowledgments}

This work was supported by the National Natural Science Foundation of China (31972546, 31501945, 31472086), Joint International Research Laboratory of Agriculture and Agri-Product Safety of the Ministry of Education of China, and the Priority Academic Program Development of Jiangsu Higher Education Institutions.

\section{Conflict of Interest}

The authors declare no conflict of interest. 


\section{References}

American Diabetes Association. Diagnosis and classification of diabetes mellitus.

Diabetes Care, 37: S81-90. 2014.

Brandt JM, Djouadi F, and Kelly DP. Fatty acids activate transcription of the muscle carnitine palmitoyltransferase I gene in cardiac myocytes via the peroxisome proliferator-activated receptor alpha. The Journal of Biological Chemistry, 273: 23786-23792. 1998 .

Buzzetti E, Pinzani $M$ and Tsochatzis EA. The multiple-hit pathogenesis of non-alcoholic fatty liver disease (NAFLD). Metabolism, 65: 1038-1048. 2016.

Cogburn LA, Trakooljul N, Wang X, Ellestad LE and Porter TE. Transcriptome analyses of liver in newly-hatched chicks during the metabolic perturbation of fasting and re-feeding reveals THRSPA as the key lipogenic transcription factor. BMC Genomics, 21: 109. 2020.

Desert C, Baéza E, Aite M, Boutin M, Cam AL, Montfort J, Houee-Bigot M, Blum Y, Roux PF, Hennequet-Antier C, Berri C, Metayer-Coustard S, Collin A, Allais S, E. Bihan L, Causeur D, Gondret F, Duclos MJ and Lagarrigue S. Multi-tissue transcriptomic study reveals the main role of liver in the chicken adaptive response to a switch in dietary energy source through the transcriptional regulation of lipogenesis. BMC Genomics, 19: 187. 2018.

Désert C, Duclos MJ, Blavy P, Lecerf F, Moreews F, Klopp C, Aubry M, Herault F, Roy PL, Berri C, Douaire M, Diot C and Lagarrigue S. Transcriptome profiling of the feeding-to-fasting transition in chicken liver. BMC Genomics, 
9: 611.2008.

Fournier E, Peresson R, Guy G and Hermier D. Relationships between storage and secretion of hepatic lipids in two breeds of geese with different susceptibility to liver steatosis. Poultry Science, 76: 599-607. 1997.

Fujita S, Yamaguchi M, Hiramoto D, Saneyasu T, Honda K and Kamisoyama H. Effects of fasting and refeeding on the mRNA levels of insulin-like growth factor-binding proteins in chick liver and brain. The Journal of Poultry Science, 55: 269-273. 2018.

Goodridge AG. Metabolism of glucose-U-14C in vitro in adipose tissue from embryonic and growing chicks. American Journal of Physiology, 214: 897-901. 1968.

Ji B, Ernest B, Gooding JR, Das S, Saxton AM, Simon J, Dupont J, Métayer-Coustard S, Campagna SR and Voy BH. Transcriptomic and metabolomic profiling of chicken adipose tissue in response to insulin neutralization and fasting. BMC Genomics, 13: 441. 2012.

Leveille GA, Romsos DR, Yeh Y and O'Hea EK. Lipid biosynthesis in the chick. A consideration of site of synthesis, influence of diet and possible regulatory mechanisms. Poultry Science, 54: 1075-1093. 1975.

Li Q, Li J, Lan H, Wang N, Hu X, Chen L and Li N. Effects of fasting and refeeding on expression of MAFbx and MuRF1 in chick skeletal muscle. Science China-Life Sciences, 54: 904-907. 2011.

Liu L, Zhao X, Wang Q, Sun X, Xia L, Wang Q, Yang B, Zhang Y, Montgomery S, 
Meng H, Geng T, and Gong D. Prosteatotic and Protective Components in a Unique Model of Fatty Liver: Gut Microbiota and Suppressed Complement System. Scientific Reports, 6: 31763. 2016.

Morais S, Knoll-Gellida A, Andre M, Barthe C and Babin PJ. Conserved expression of alternative splicing variants of peroxisomal acyl-CoA oxidase 1 in vertebrates and developmental and nutritional regulation in fish. Physiological Genomics, 28: 239-252. 2007.

Navidshad B and Royan M. Ligands and regulatory modes of peroxisome proliferator-activated receptor gamma (PPAR $\gamma)$ in avians. Critical Reviews in Eukaryotic Gene Expression, 25: 287-292. 2015.

O'Hea EK and Leveille GA. Lipid biosynthesis and transport in the domestic chick (Gallus domesticus). Comparative Biochemistry and Physiology, 30: 149-159. 1969.

Rakhshandehroo M, Sanderson LM, Matilainen M, Stienstra R, Carlberg C, Groot PJ, Muller M and Kersten S. Comprehensive analysis of PPARalpha-dependent regulation of hepatic lipid metabolism by expression profiling. PPAR Research, 2007: 26839. 2007.

Shchelkunova TA, Morozov IA, Rubtsov PM, Bobryshev YV, Sobenin IA, Orekhov AN, Andrianova IV and Smirnov AN. Lipid regulators during atherogenesis: expression of LXR, PPAR, and SREBP mRNA in the human aorta. PLoS One, 8: e63374. 2013.

Streba LA, Carstea D, Mitrut P, Vere CC, Dragomir N and Streba CT. Nonalcoholic 
fatty liver disease and metabolic syndrome: a concise review. Romanian Journal of Morphology and Embryology, 49: 13-20. 2008.

Wang Y, Buyse J, Courousse N, Tesseraud S, Métayer-Coustard S, Berri C, Schallier $\mathrm{S}$, Everaert $\mathrm{N}$ and Collin A. Effects of sex and fasting/refeeding on hepatic AMPK signaling in chickens (Gallus gallus). Comparative Biochemistry and Physiology A-Molecular and Integrative Physiology, 240: 110606. 2020.

Yeh SJ and Leveille GA. Significance of skin as a site of fatty acid and cholesterol synthesis in the chick. Proceedings of the Society for Experimental Biology and Medicine, 142: 115-119. 1973.

Yeon JE, Choi KM, Baik SH, Kim KO, Lim HJ, Park KH, Kim JY, Park JJ, Kim JS, Bak YT, Byun KS and Lee $\mathrm{CH}$. Reduced expression of peroxisome proliferator-activated receptor-alpha may have an important role in the development of non-alcoholic fatty liver disease. Journal of Gastroenterology and Hepatology, 19: 799-804. 2004. 


\section{Legends to figures}

Fig. 1. The top 20 KEGG pathways enriched with differentially expressed genes identified in the fasting vs. control groups (A) or in the refeeding vs. fasting groups (B) of geese. The $\mathrm{x}$-axis shows the enrichment factor and the $\mathrm{y}$-axis indicates the associated KEGG pathways. The sizes of the dots reflect the numbers of differentially expressed genes associated with each pathway, and the colors of the dots indicate the q-values; the smaller the q-value, the more significant the enrichment.

Fig. 2. Validation of selected differentially expressed genes in the livers of control, fasted, and refed geese determined by quantitative reverse transcription-PCR (qRT-PCR) with GAPDH as the reference gene. (A) The expression level is presented as the fold change relative to the control group $(\mathrm{n}=8) ;{ }^{*} \mathrm{p}<0.05$ and ${ }^{* *} \mathrm{p}<$ 0.01. The data are expressed as means \pm SE. (B) Comparisons of gene expression levels between transcriptome analysis and qRT-PCR analysis based on the fold change of gene expression. " $r$ " denotes the Pearson correlation coefficient and ** denotes $\mathrm{p}<0.01$. 
Table 1. Primer sequences for qPCR analysis.

\begin{tabular}{lll}
\hline Gene & Forward $\left(5^{\prime}-3^{\prime}\right)$ & Reverse $\left(5^{\prime}-3^{\prime}\right)$ \\
\hline ACSBG2 & TACCTCCTCCCTCCAGTGTG & GCTTTATCCACTGGCCACCT \\
ACOX1 & AGCCGCTGGATCTTCATCTG & GTGGGGCTGTTGAGGATGAA \\
CPT1A & GCATTGACCGCCATCTGTTC & GCCAGCATCTCAGGGTTCTT \\
ACSL5 & TTCCTCCCGCTGACTTGAAC & TCCAGAAGCATGCAGTCCTC \\
ACAA1 & AAGCCAGGCTGTGTACTGTG & CCATGCCAGTTCCAATGCAC \\
CPT1B & CTTCACCCTGCCCACTGTAG & CCCGTGGGTTGATGTTCTGA \\
EHHADH & ACTCAGCGACTACCCAGACT & TTTGCCAAGCGAATTCCTGC \\
SCD & CTTCACCCTGCCCACTGTAG & CCCGTGGGTTGATGTTCTGA \\
PCK1 & TTACCCAGGGGGATCTGGAG & AGAGCCAACCAGCAGTTCTC \\
GAPDH & CTGATGCTCCCATGTTCGTG & CCACGATGCCAAAGTTGTCA \\
\hline & GCACCCAGCACGATGAAAAT & GACAATGGAGGGTCCGGATT \\
\hline
\end{tabular}


Table 2. Top 10 up/down-regulated differentially expressed genes between the fasting vs. control groups.

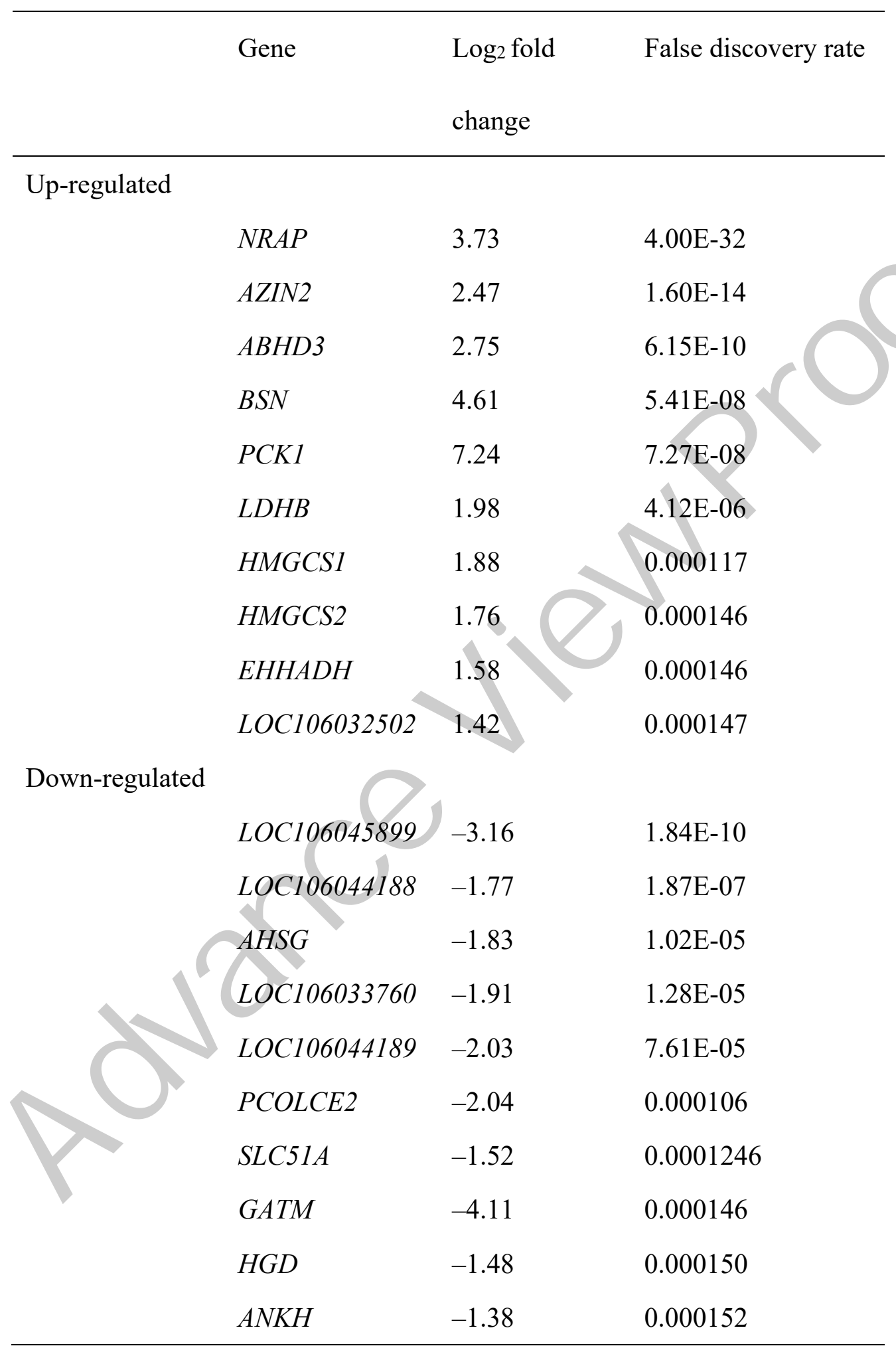


Table 3. Top 10 up/down-regulated differentially expressed genes between the refeeding vs. fasting groups.

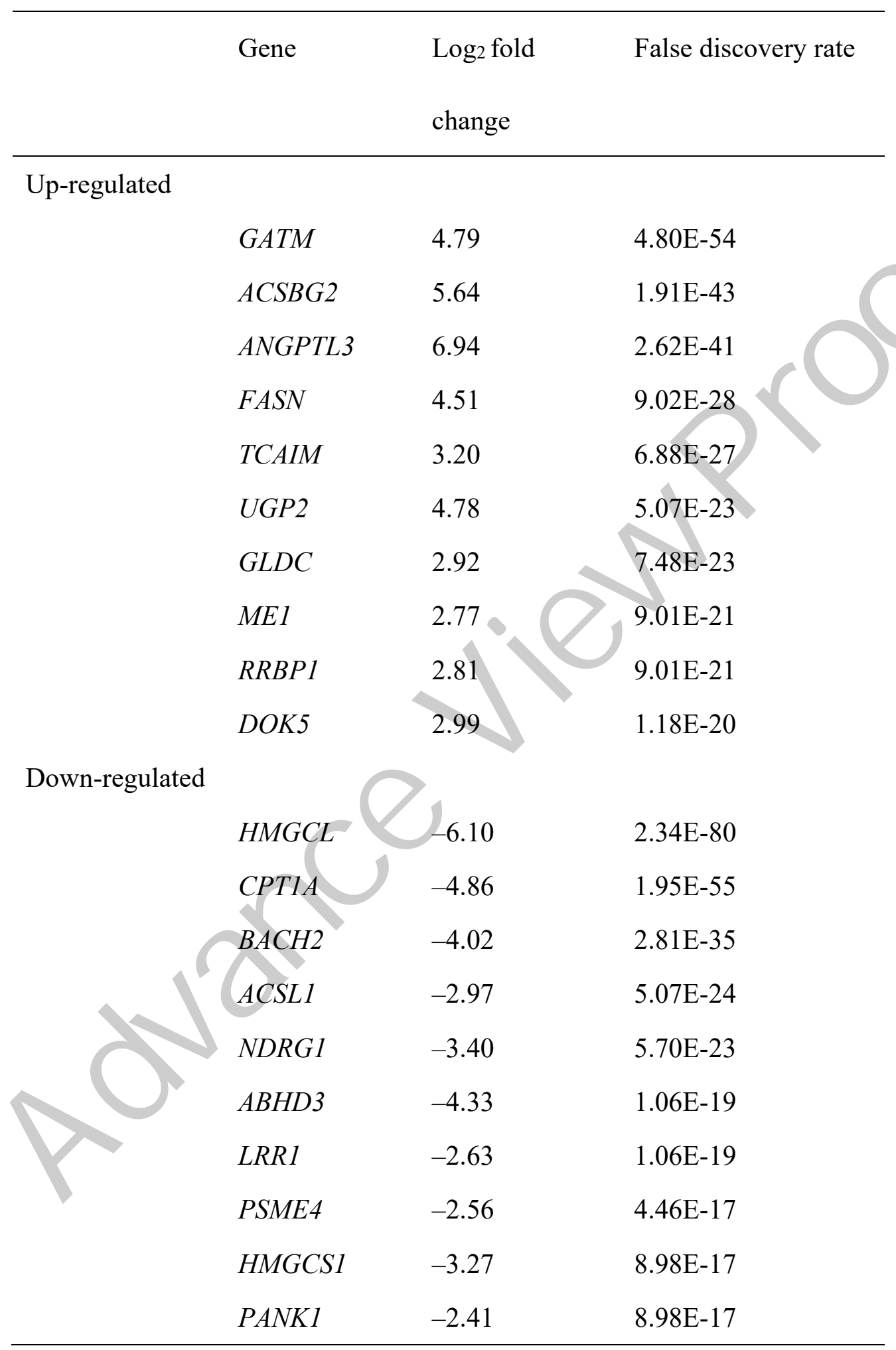




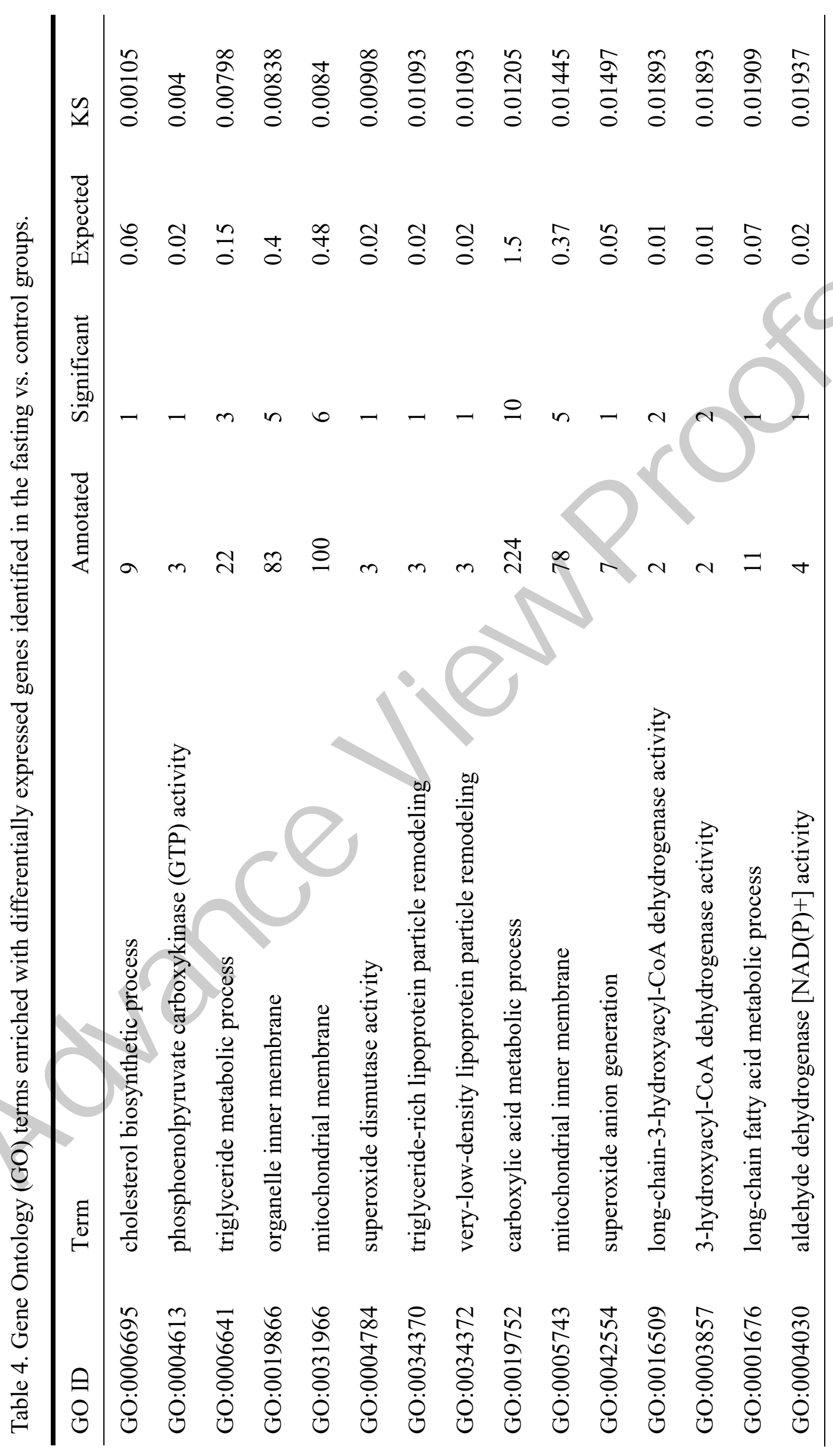




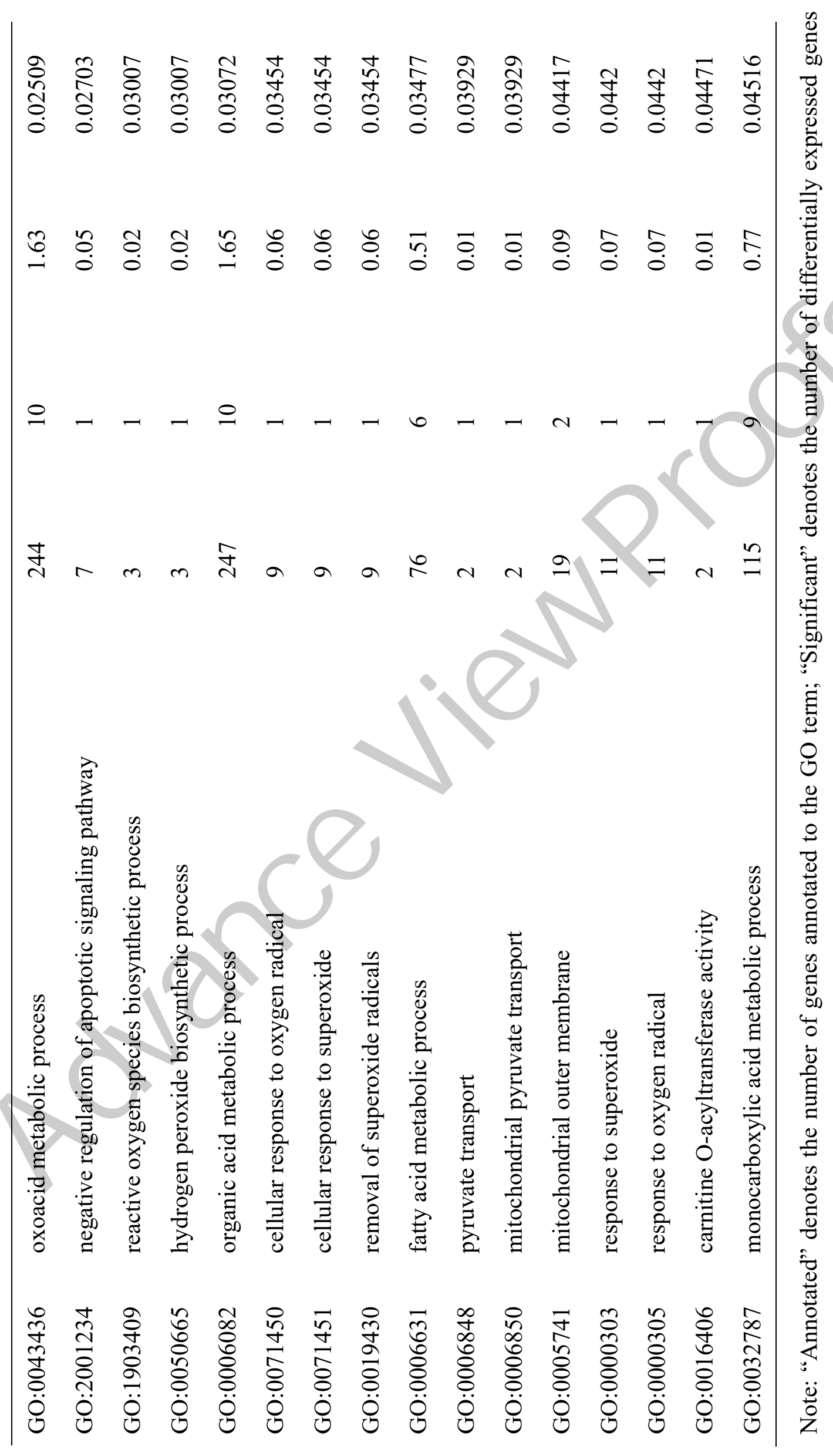




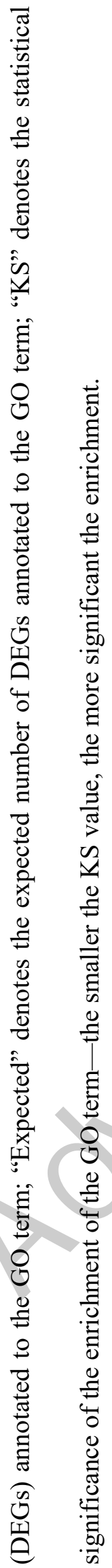




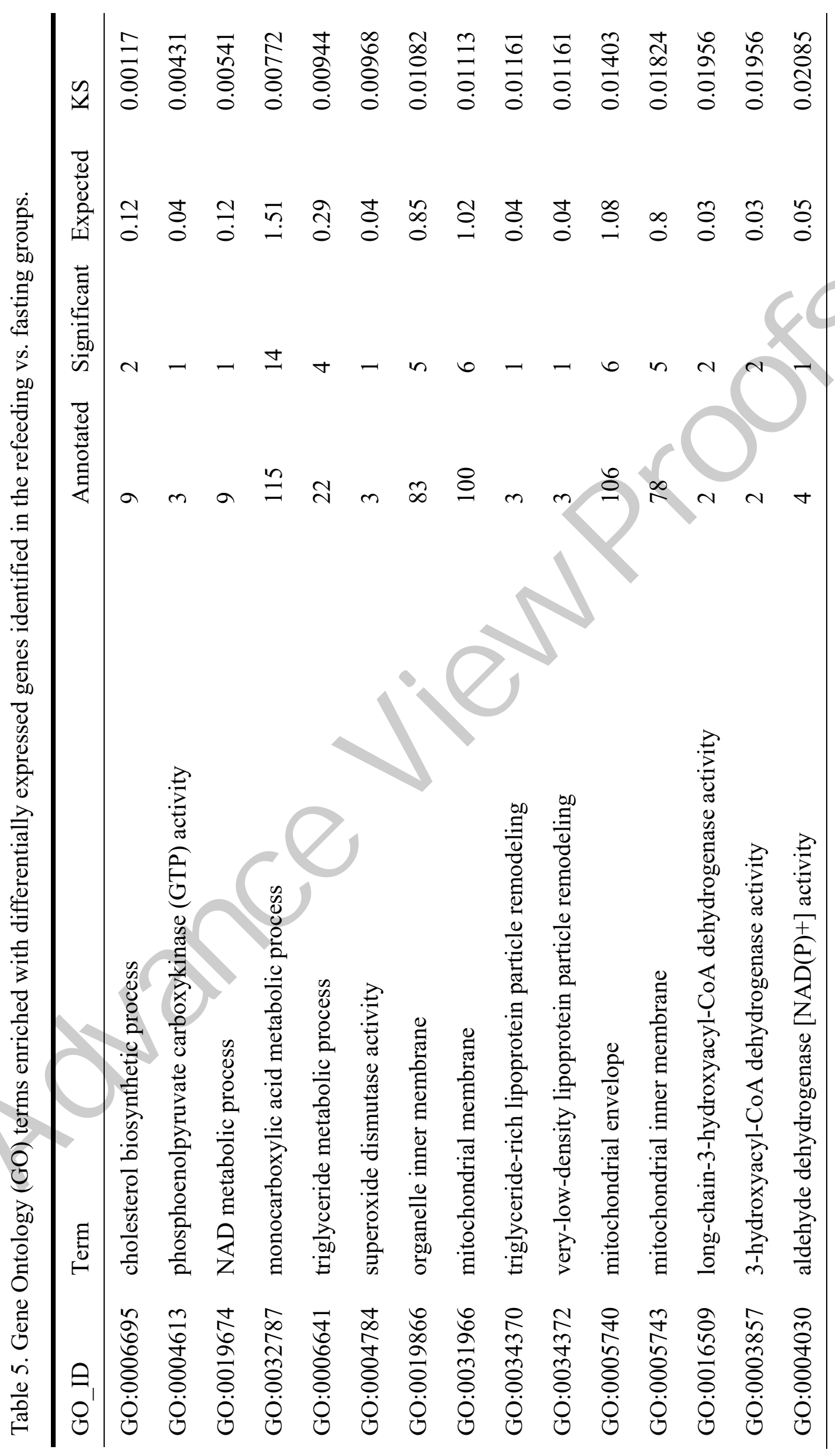




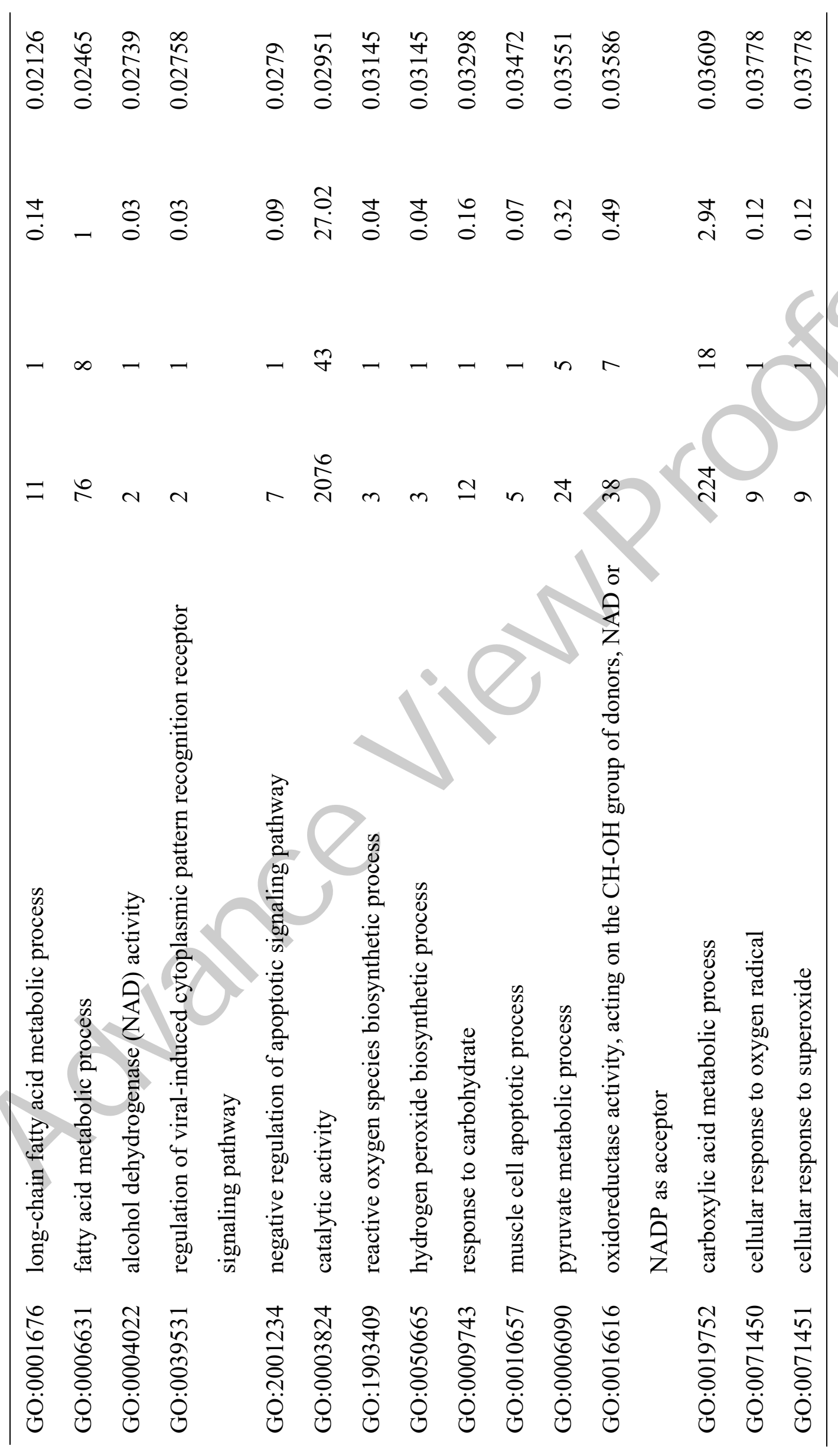




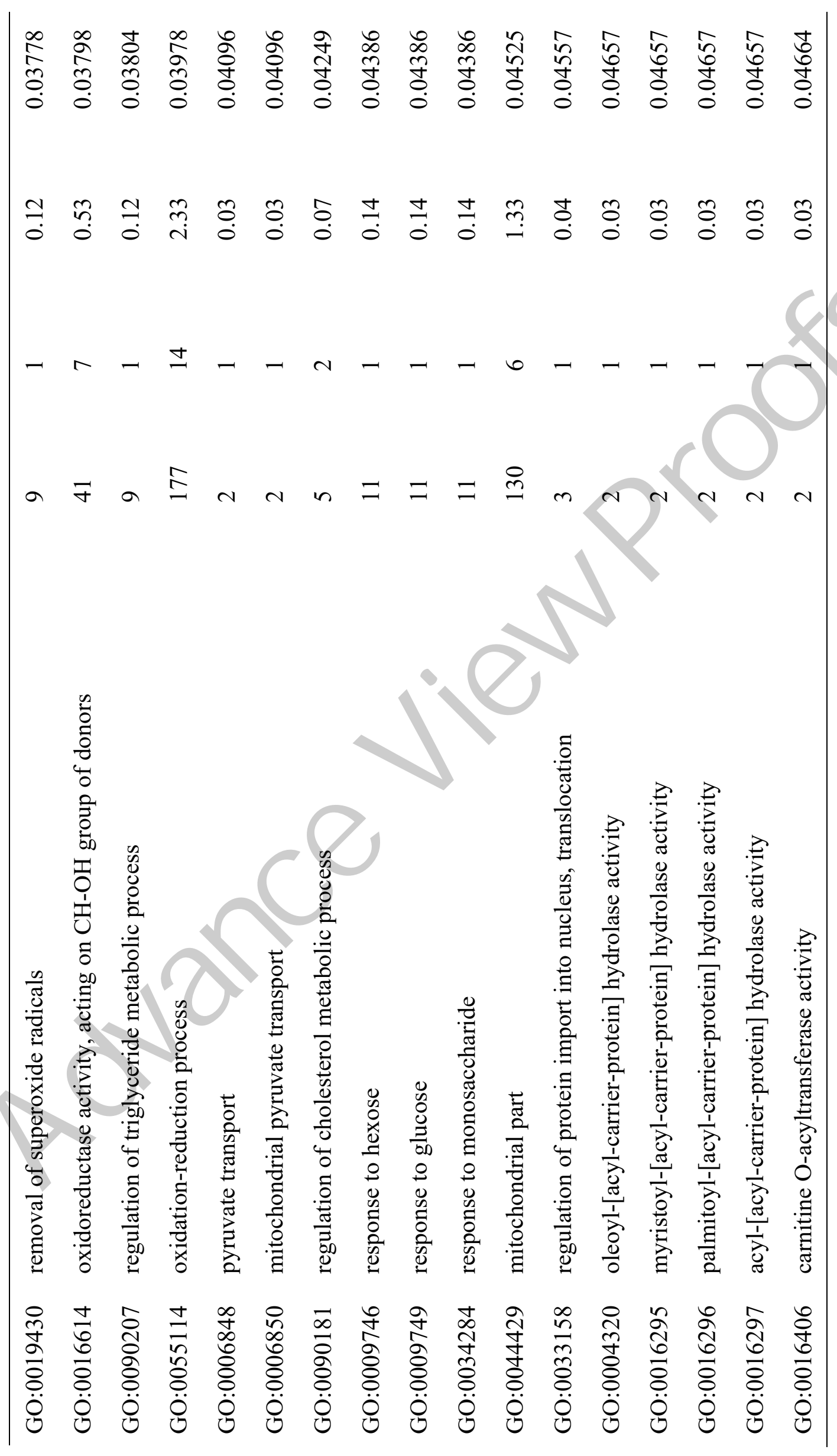




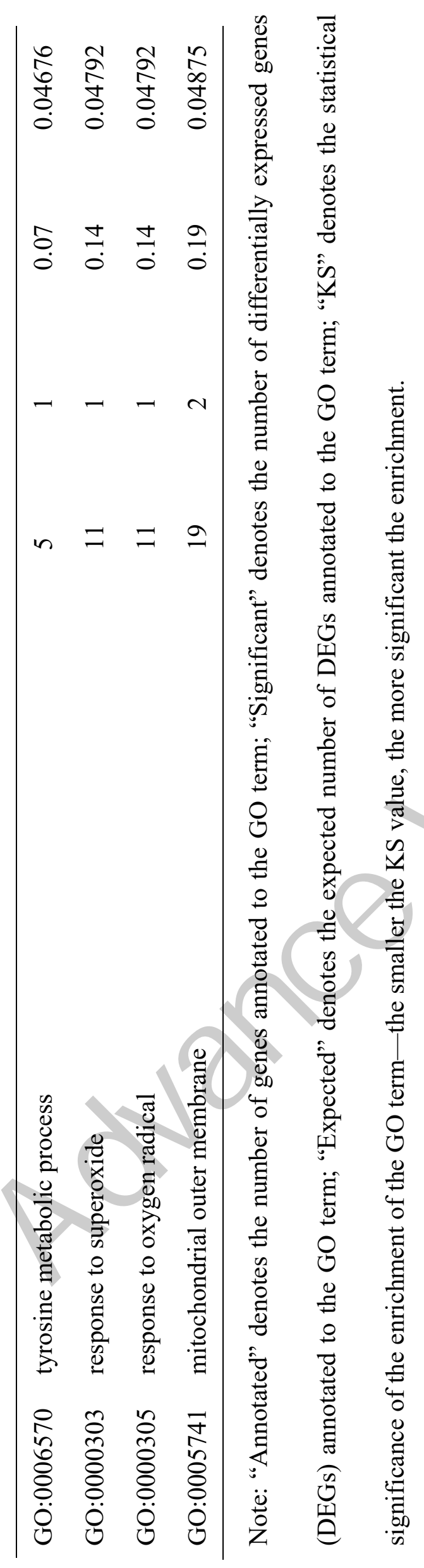




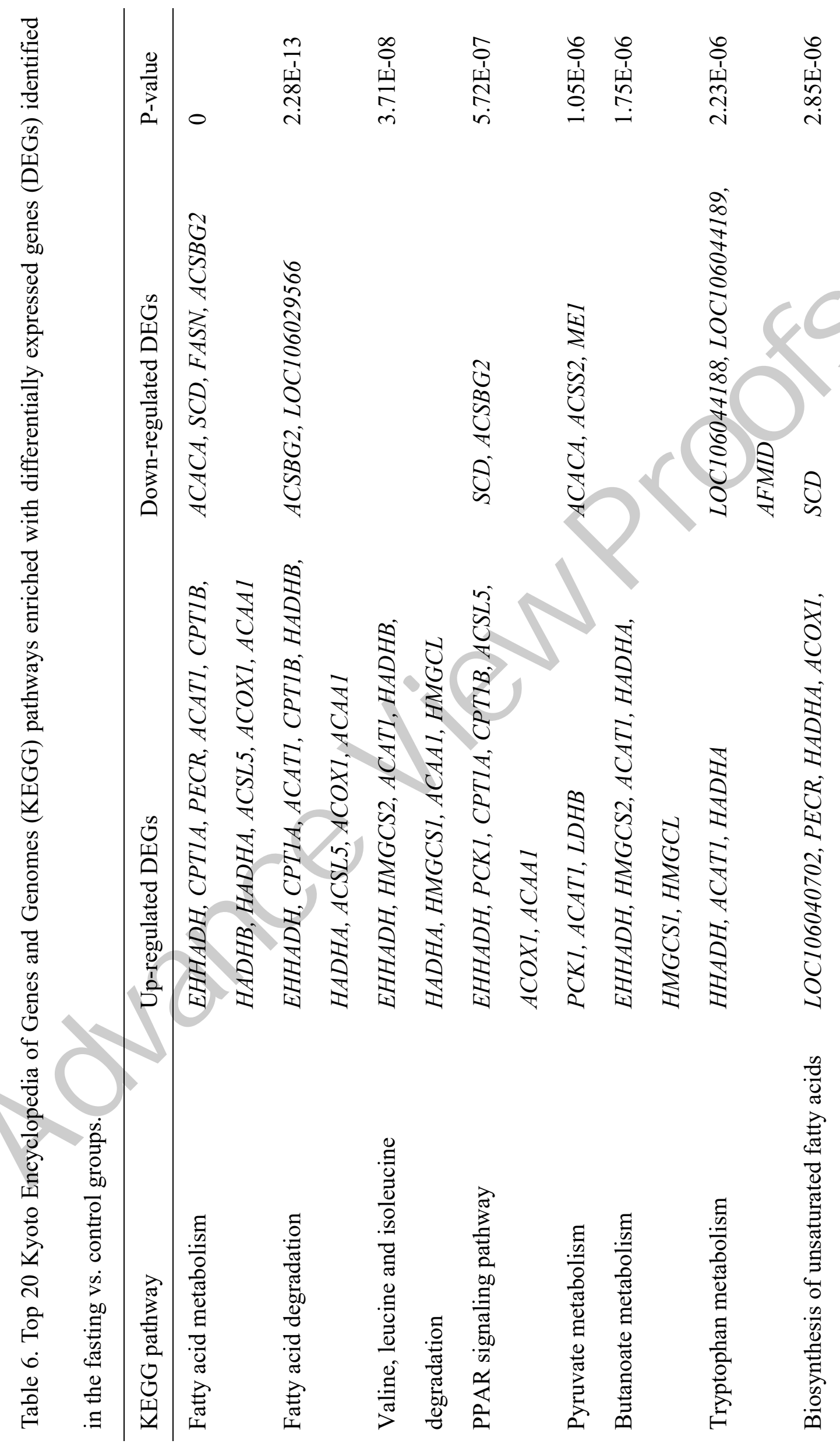




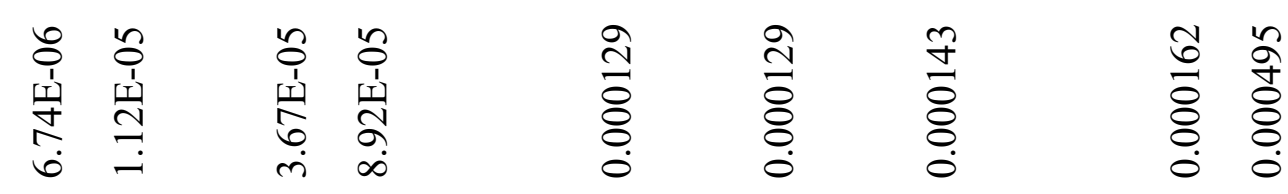
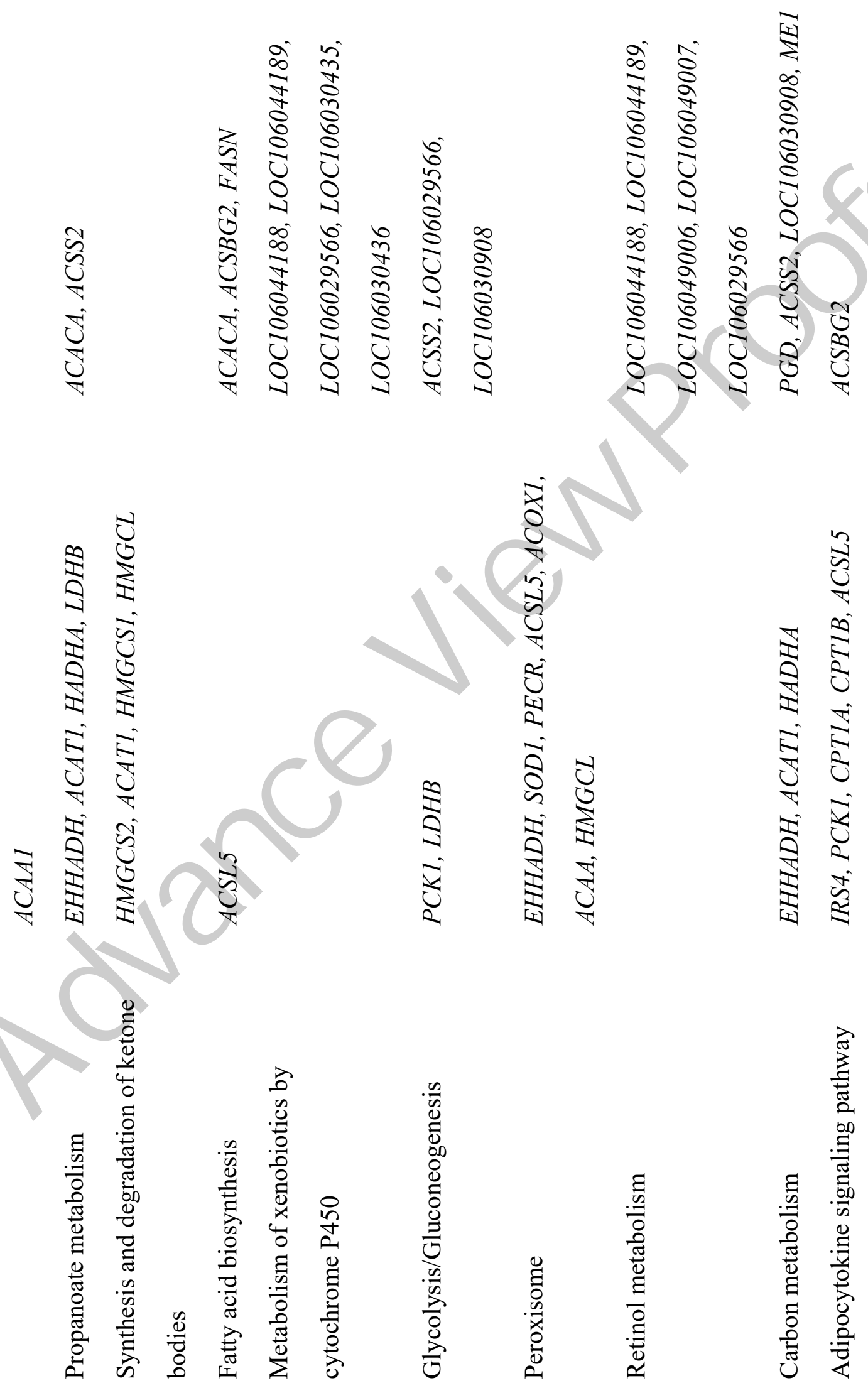


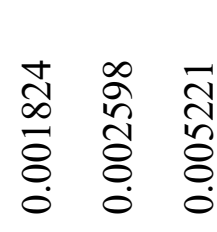

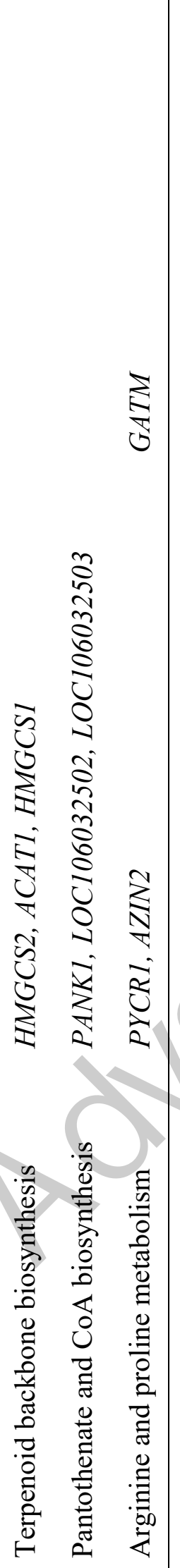




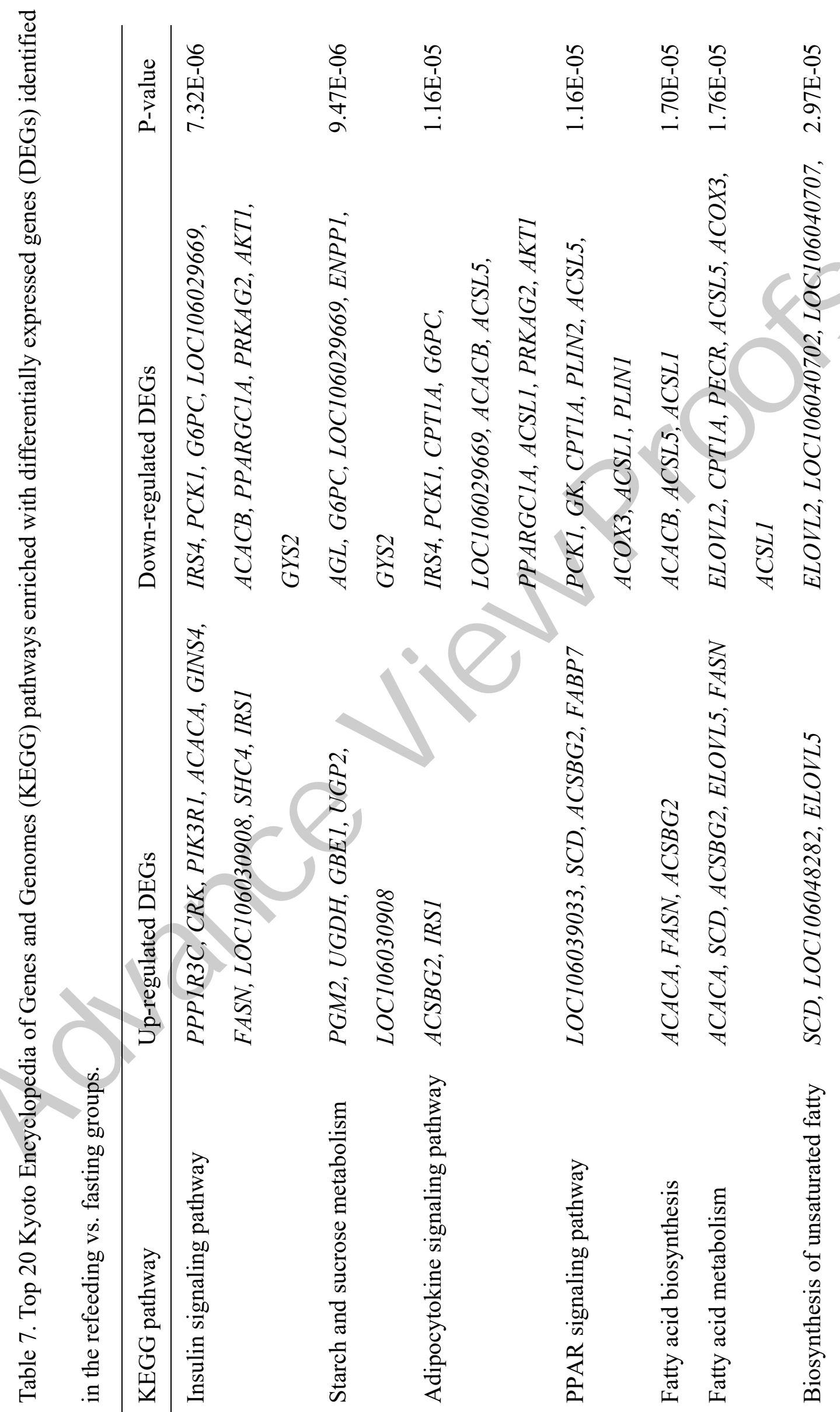




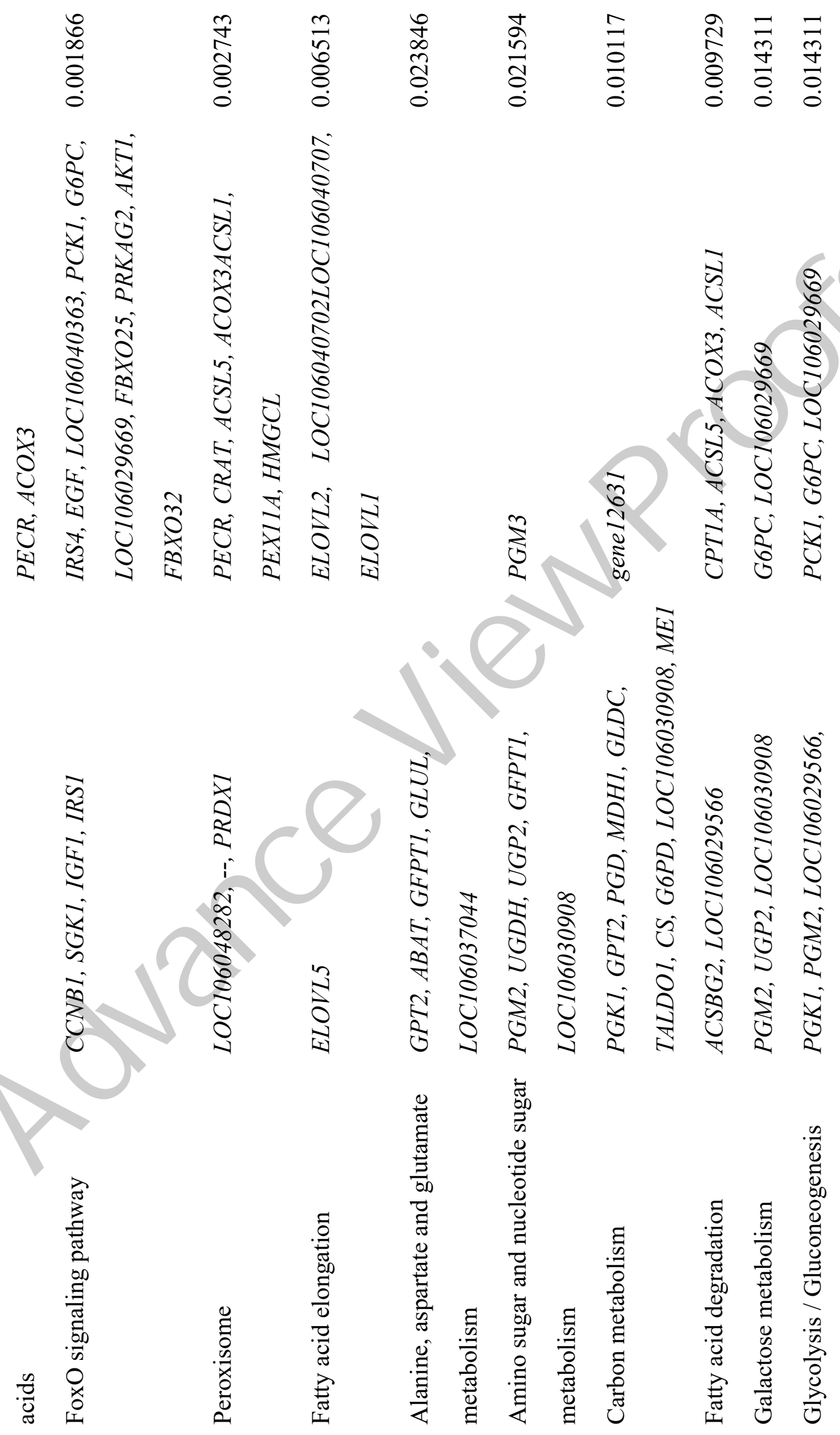




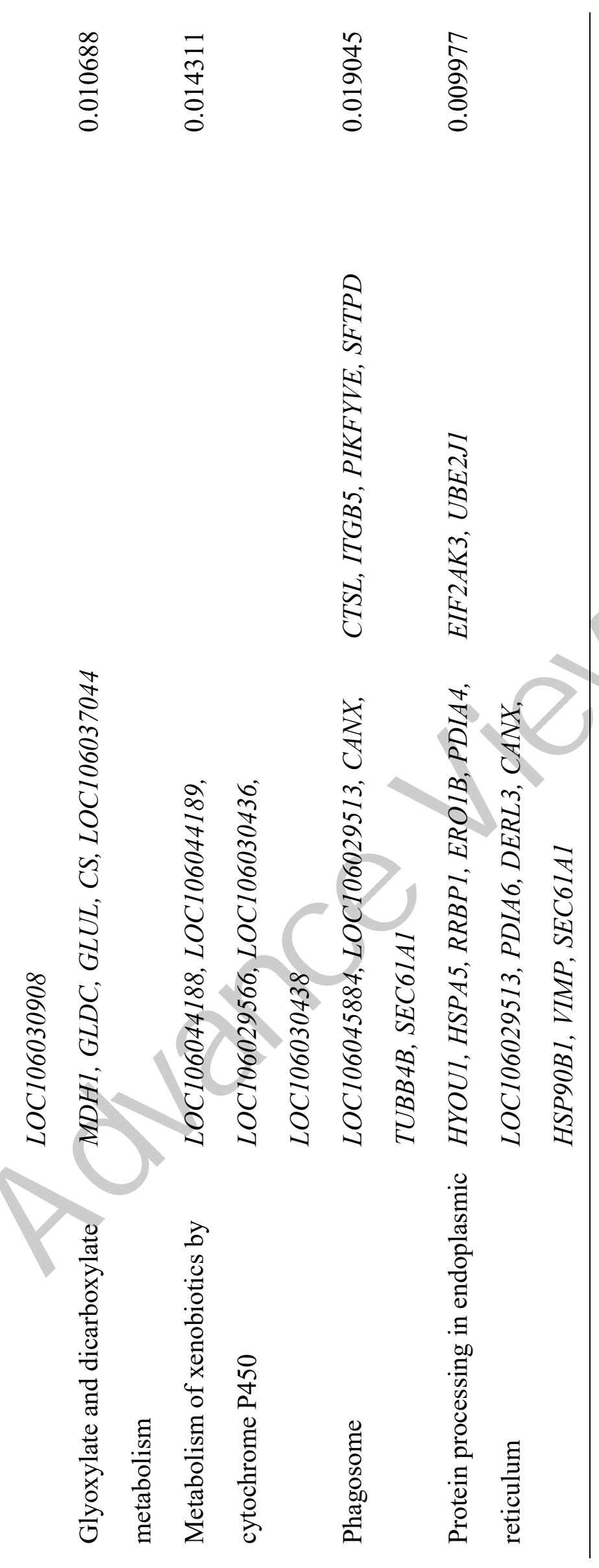




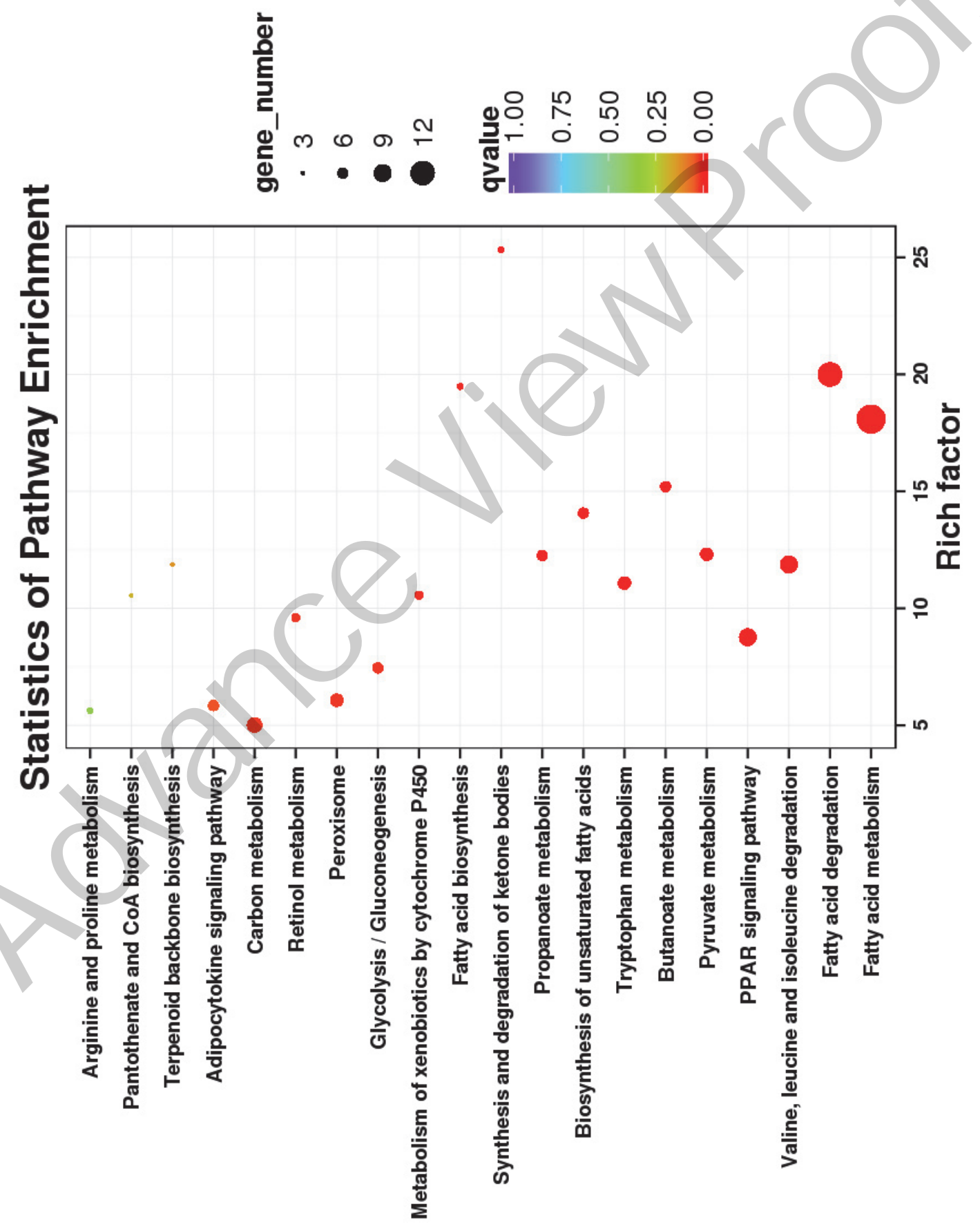




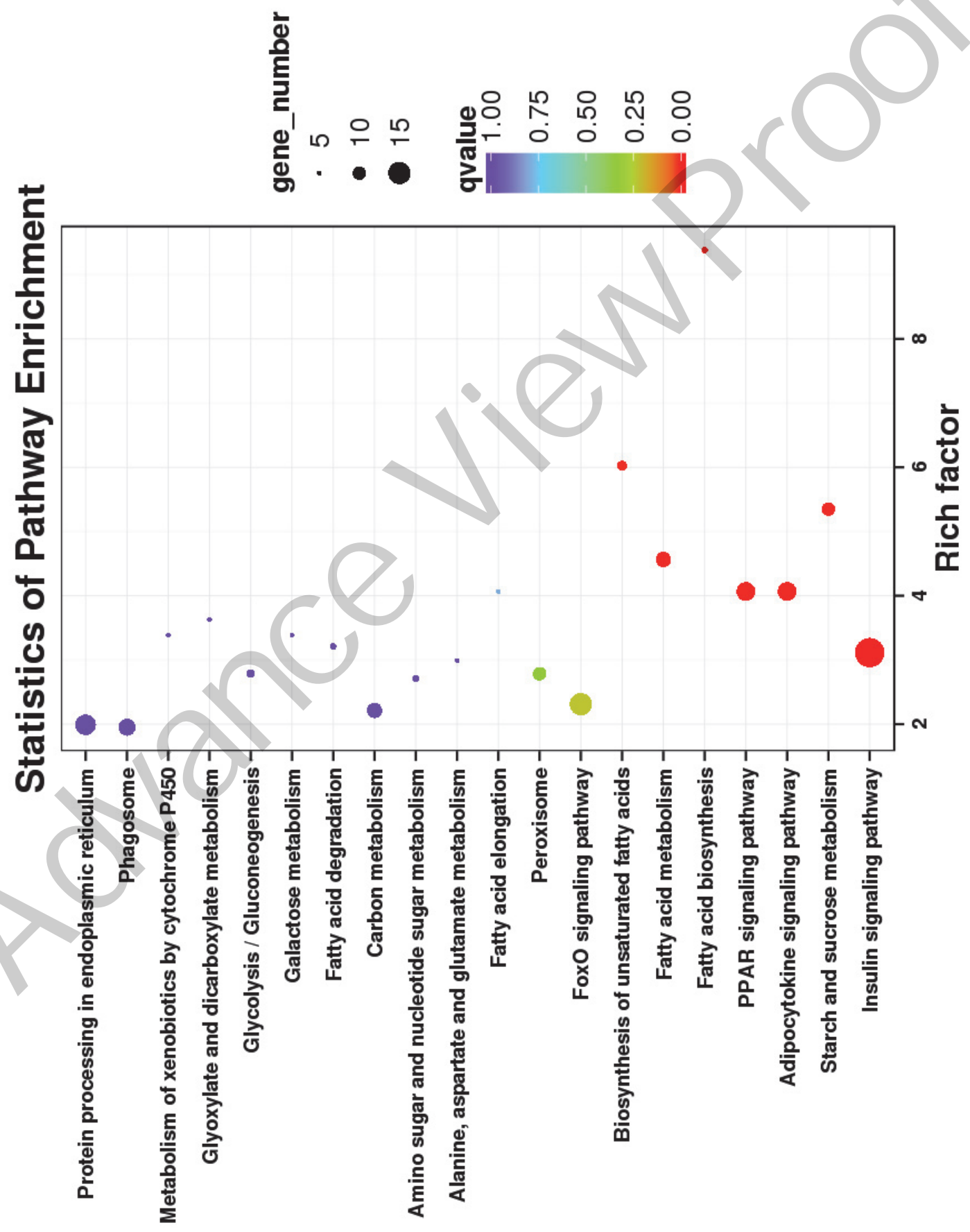



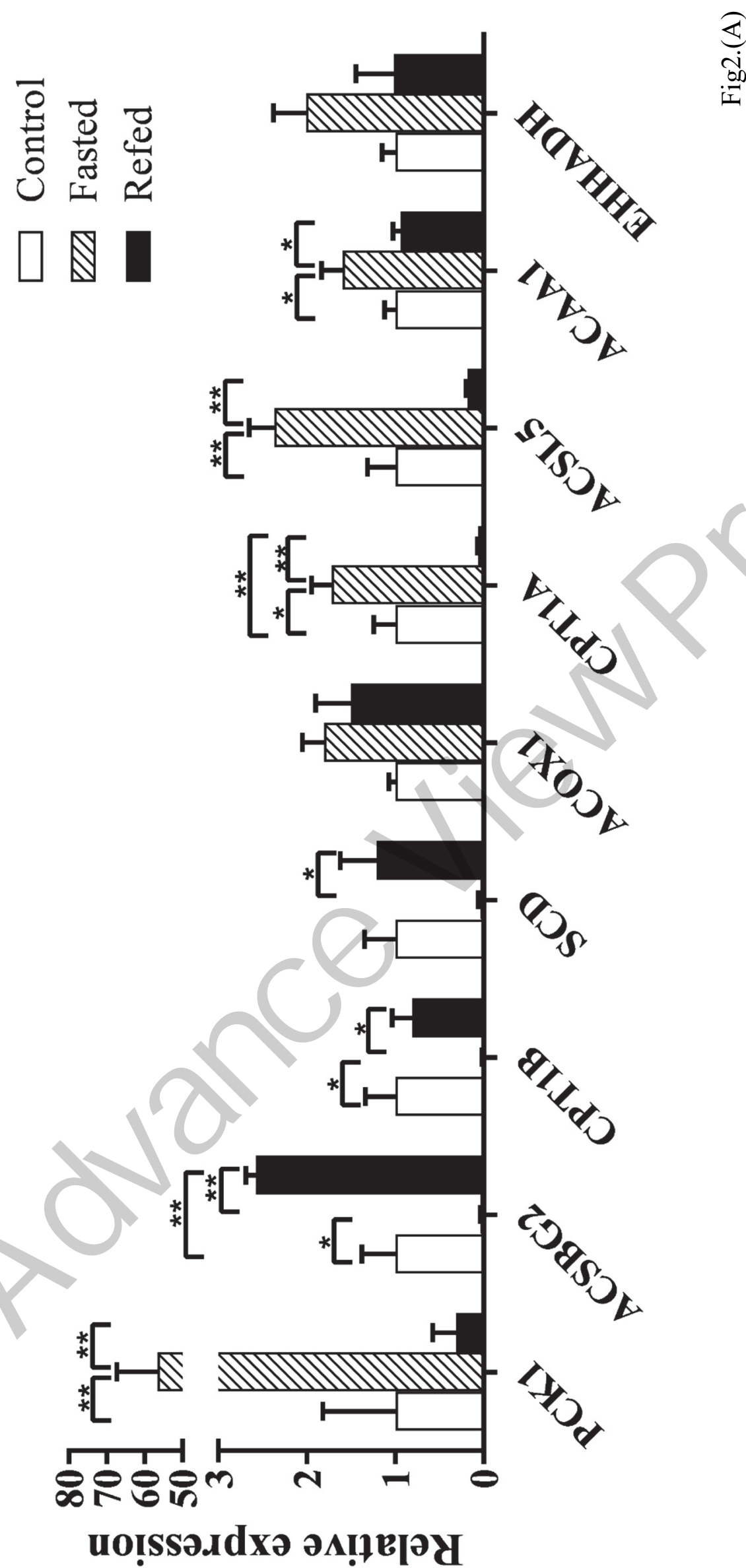


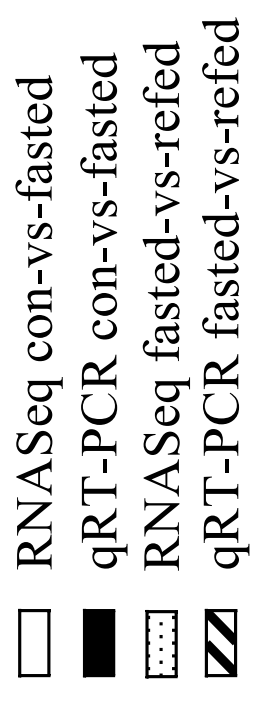

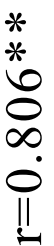
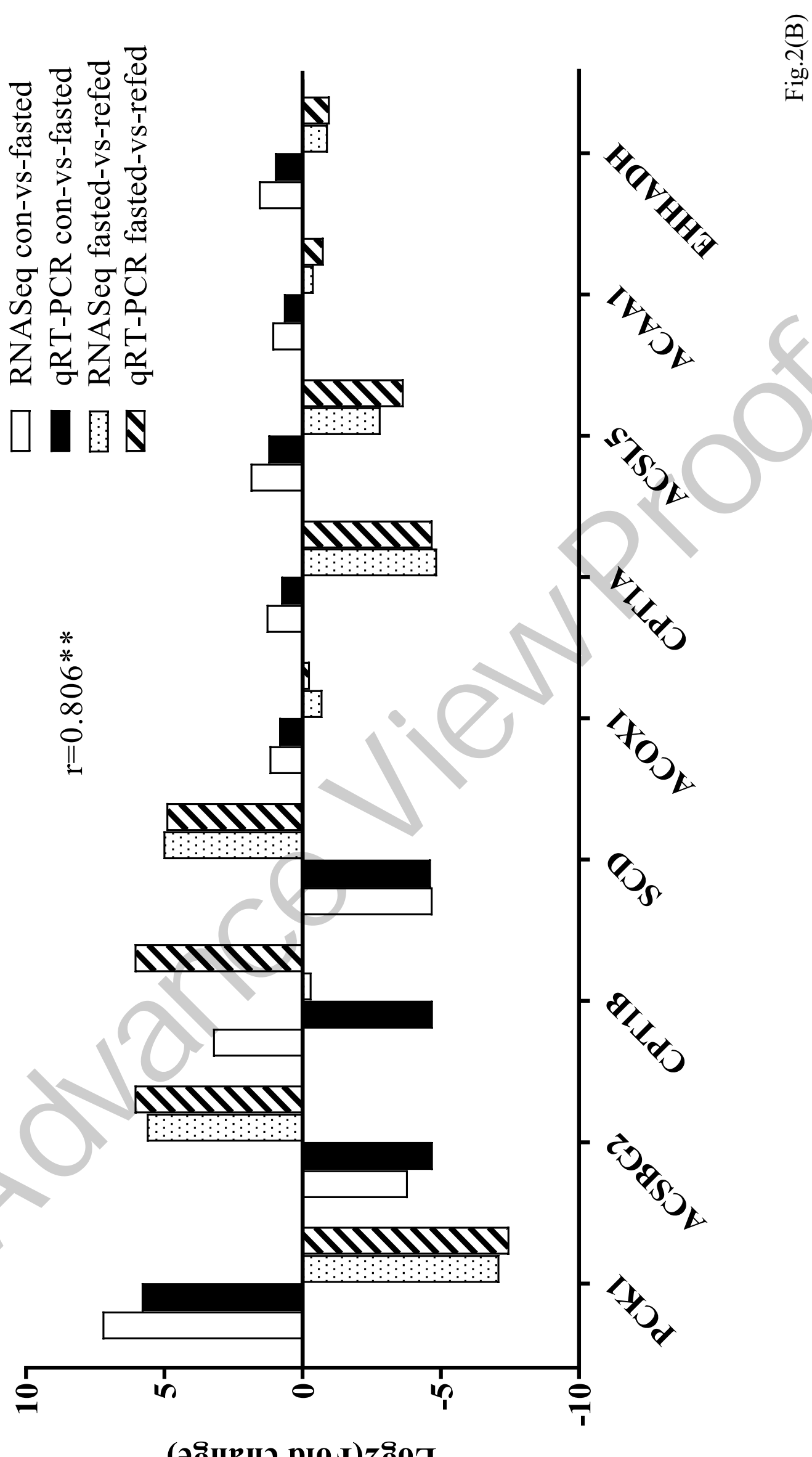

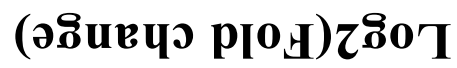

